\title{
ACCURATE TIME/FREQUENCY TRANSFER METHOD USING BI-DIRECTIONAL WDM TRANSMISSION
}

\author{
Atsushi Imaoka and Masami Kihara \\ NTT Optical Network Systems Laboratories \\ 1-2356 Take, Yokosuka, Kanagawa, 238-03, Japan
}

\begin{abstract}
An accurate time transfer method is proposed using bi-directional wavelength division multiplexing (WDM) signal transmission along a single optical fiber. This method will be used in digital telecommunication networks and yield a time synchronization accuracy of better than $1 \mathrm{~ns}$ for long transmission lines over several tens of kilometers. The method can accurately measure the difference in delay between two wavelength signals caused by the chromatic dispersion of the fiber in conventional simple bi-directional dual-wavelength frequency transfer methods. We describe the characteristics of this difference in delay and then show that the accuracy of the delay measurement can be obtained below $0.1 \mathrm{~ns}$ by transmitting $156 \mathrm{Mb} / \mathrm{s}$ time reference signals of 1.31 un and 1.55 Im along a $50 \mathrm{~km}$ fiber using the proposed method. The sub-nanosecond delay measurement using the simple bi-directional dual-wavelength transmission along a $100 \mathrm{~km}$ fiber with a wavelength spacing of $1 \mathrm{~nm}$ in the $1.55 \mu \mathrm{m}$ range is also shown.
\end{abstract}

\section{INTRODUCTION}

In digital telecommunication networks, a reference frequency is already being distributed to network nodes in order to synchronize digital switching and multiplexing equipment. If the transmission delay is compensated for by measuring the round-trip path delay using the outgoing and incoming paths, accurate time transfer over long distances will be achieved. The proposed method will yield a time synchronization accuracy of better than 1 ns for long transmission lines over several tens of kilometers. This is useful for operating a distribution network, for locating faults, and in several network time services.

Accuracy in time transfer is determined by the accuracy to which the propagation delay is measured. Since the round-trip path is comprised of paired physically-independent fibers in conventional communication systems[1], the delay measurement accuracy is limited by the asymmetry of the fiber length. A delay-measuring system using simple bi-directional dualwavelength transmission along a single fiber ${ }^{[2]}$ eliminates fiber-length asymmetry, but it incurs error due to the chromatic dispersion of the fiber.

This paper proposes a novel delay-measuring system using bi-directional WDM transmission along a single optical fiber, which can eliminate the error caused by fiber dispersion. The effects 
from the difference in delay between the two wavelength signals and the variations are described. The $1.31 \mu \mathrm{m}$ and $1.55 \mu \mathrm{m}$ signal transmissions along a $50 \mathrm{~km}$ fiber are tested and the feasibility of sub-nanosecond time transfer using the proposed method is confirmed. The bi-directional dual-wavelength transmission along a $100 \mathrm{~km}$ fiber is also shown, with a wavelength spacing of $1 \mathrm{~nm}$ in the $1.55 \mu \mathrm{m}$ range. In such a close wavelength transmission system, measuring the sub-nanosecond delay is possible using a simple bi-directional dual-wavelength transmission method (i.e., not using the proposed method).

\section{DELAY MEASURING METHOD}

Simple bi-directional dual-wavelength optical transmission for transferring time signals is shown in Figure 1. The master and slave nodes are connected with a single optical fiber and wavelength division multiplexers/demultiplexers. The reference time signals are generated by the reference clock installed in the master node and transmitted from the master to the slave node using $\lambda_{1}$ wavelength light. This $\lambda_{1}$ light is modulated using a digital data stream which includes time signals. The feedback time signals are transferred from the slave to the master node using $\lambda_{2}$ wavelength light. The time interval counter continuously measures the round-trip delay, $\tau_{\text {sum }}$, which is the sum of $\lambda_{1}$ propagation delay, $\tau_{1}$, and $\lambda_{2}$ delay, $\tau_{2}$. The $\lambda_{1}$ propagation delay is estimated as half of $\tau_{\text {sum }}$ in this scheme. The delay information is sent to the slave node. In this node, the reference time signal derived from the $\lambda_{1}$ light is advanced by electrical phase compensation techniques according to the received delay information.

In this method, the delay measurement error is due to the difference in delay between two wavelength signals, which is caused by the chromatic dispersion of the optical fiber. The proposed delay measurement scheme, presented in Figure 2, compensates for the error due to this fiber dispersion. The reference time signals are always transmitted from the master to the slave using the $\lambda_{1}$ wavelength light. The two $1 \times 2$ optical switches change the transfer direction of the $\lambda_{2}$ wavelength light which transfers the probe time signals. When $\lambda_{2}$ signals are transferred from the slave to the master, the time interval counter $\left(\mathrm{TI}_{1}\right)$ measures the round-trip signal delay, $\tau_{\text {sum }}$. The difference in delay, $\tau_{\text {diff }}$, equals $\tau_{2}-\tau_{t} 1$, which can be measured by transferring the $\lambda_{2}$ signals from the master to the slave in the same direction as the $\lambda_{1}$ signals. The $\lambda_{1}$ propagation delay can be accurately determined from the following relationship by measuring both the sum and difference terms.

$$
\tau_{1}=\left(\tau_{\text {sum }}-\tau_{\text {diess }}\right) / 2
$$

The optical fiber delays, $t a u_{1}$ and $\tau_{2}$, vary with changes in the environment, especially temperature. Hence, both $\tau_{\text {sum }}$ and $\tau_{\text {diff }}$ must be measured repeatedly. The measurement interval required to accurately transfer time depends on the characteristics of the variations in $\tau_{1}$ and $\tau_{2}$. They are discussed in the following section. 


\section{EFFECT OF DISPERSION}

The difference in delay between two wavelength signals is caused by the difference in the group refractive index between the two wavelengths. The chromatic dispersion, $D$, is the wavelength variation coefficient of the group refractive index and is illustrated in Figure 3 . The characteristic parameters of the dispersion are zero-dispersion wavelength, $\lambda_{0}$, and the dispersion slope at zero-dispersion wavelength, $S_{0}$. The hatched area shown in Figure 3 expresses the difference in delay between two wavelengths, $\lambda_{1}$ and $\lambda_{2}$.

We consider here the difference in delay, $\tau_{\text {diff }}$, and its variation, $\Delta \tau_{\text {diff }}$. This variation, $\Delta \tau_{\text {diff }}$, is caused by the difference in the change of the fiber dispersion characteristics with temperature between two wavelength signals. Assuming that $S_{0}$ is constant against temperature change in the range from $\lambda_{1}$ to $\lambda_{2}$ and that the zero-dispersion wavelength is only shifted by temperature change, $\tau_{\text {diff }}$ and $\Delta \tau_{\text {diff }}$ with temperature are

$$
\begin{aligned}
\tau_{\text {diff }} & =\frac{1}{2} S_{O} \cdot\left(\left(\lambda_{2}-\lambda_{0}\right)^{2}-\left(\lambda_{1}-\lambda_{0}\right) 62\right) \cdot L \\
\Delta \tau_{d i f f} & =S_{O} \cdot\left(\lambda_{1}-\lambda_{2}\right) \cdot \frac{d \lambda_{0}}{d T} \cdot L .
\end{aligned}
$$

where $L, \Delta T$, and $d \lambda_{0} / d T$ denote the fiber length, temperature change, and the temperature dependence of zero-dispersion wavelength, respectively.

The normal single mode fiber (SMF) has a zero-dispersion wavelength around $1310 \mathrm{~nm}$. The dispersion shifted fiber (DSF) has a $\lambda_{0}$ around $1550 \mathrm{~nm}$ and is optimized for transmission in the $1550 \mathrm{~nm}$ wavelength region. A typical value of the dispersion slope is about $+0.07 \mathrm{ps} / \mathrm{nm}^{2} / \mathrm{km}$. It is reported that the temperature dependence of the zero-dispersion wavelength, $d \lambda_{0} / d T$, is about $+0.03 \mathrm{~nm} /{ }^{\circ} \mathrm{C}[3]$. Figure 4 shows the difference in delay per unit fiber length, $\tau_{\text {diff }} / L$, as a function of the wavelength difference $\Delta \lambda_{0}\left(=\lambda_{1}-\lambda_{2}\right)$ for three different zero-dispersion wavelength fibers. In this calculation, the above parameters and $\lambda_{1}=1550 \mathrm{~nm}$ were used. The zero-dispersion wavelength of $1310 \mathrm{~nm}$ denotes the system using the SMF. The $\lambda_{0}=1550 \mathrm{~nm}$ and $\lambda_{0}=1600 \mathrm{~nm}$ are the best and worst cases using the $1.55 \mu \mathrm{m}$ DSF, respectively, because the zero-dispersion wavelength of the DSF is specified in the region from 1500 to $1600 \mathrm{~nm}^{[4]}$.

As shown in Figure 4, $\tau_{\text {diff }} / L$ becomes $2.0 \mathrm{~ns} / \mathrm{km}$ in the system using $1550 \mathrm{~nm}$ and $1310 \mathrm{~nm}$ as dual-wavelength signals, where $\lambda_{0}=1550 \mathrm{~nm}$. For example, the $\tau_{\text {diff }}$ becomes $100 \mathrm{~ns}$ where $\mathrm{L}$ $=50 \mathrm{~km}$ and it must be compensated for by using our proposed scheme described in the above section for ensuring sub-nanosecond time transfer. Where $\Delta T=40^{\circ} \mathrm{C}$, the $\Delta \tau_{\text {diff }}$ becomes 1.0 ns, thus, $\tau_{\text {diff }}$ must be measured frequently according to the temperature change by changing the direction of the $\lambda_{2}$ signals in the proposed method.

If two close wavelength signals around $1550 \mathrm{~nm}$ are used, the difference in delay and its variation become small. For example, where $\lambda_{0}=1600 \mathrm{~nm}, \Delta \lambda=1 \mathrm{~nm}, \mathrm{~L}=100 \mathrm{~km}$, and

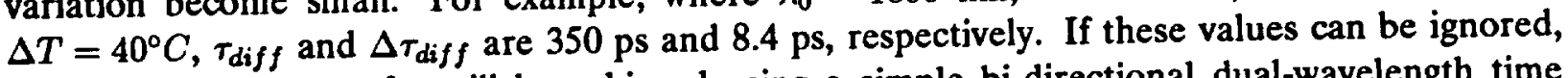
an accurate time transfer will be achieved using a simple bi-directional dual-wavelength time transfer scheme in which two wavelength signals are close. The system using close wavelength 
signals within the $1.55 \mu \mathrm{m}$ band has several advantages compared to the 1.31 and $1.55 \mu \mathrm{m}$ WDM systems: the fiber span can be wider, higher bit-rate signals can be transmitted, and the amount of short-term jitter is expected to be smaller. Recently, many wavelength multiplexing devices such as the arrayed waveguide grating[5] and the fiber grating filter ${ }^{[6]}$ have been developed for the $1.55 \mu \mathrm{m}$ multi-wavelength division multiplexing system. Therefore, such close wavelength multiplexing and demultiplexing can be easily performed.

\section{EXPERIMENTAL RESULTS}

\section{$1.31 \mu \mathrm{m}+1.55 \mu \mathrm{m}$ WDM Transmission}

Two $155.52 \mathrm{Mb} / \mathrm{s}$ timing reference signals were transmitted bi-directionally along a $50 \mathrm{~km} 1.55$ $\mu \mathrm{m}$ dispersion shifted fiber (DSF). The DSF had a loss of $0.2 \mathrm{~dB} / \mathrm{km}$ and a zero dispersion at the $1556 \mathrm{~nm}$ wavelength. Fabry-Perot multi-mode laser diodes (FP-LDs) operating at $1548 \mathrm{~nm}$ and $1315 \mathrm{~nm}$ were used as the $\lambda_{1}$ and $\lambda_{2}$ light sources with the respective output power of -0.4 $\mathrm{dBm}$ and $+1.8 \mathrm{dBm}$. Both FP-LDs were directly modulated by $155.52 \mathrm{Mb} / \mathrm{s}$ digital signals that included time signals of one pulse per second. The details of the digital signals including time signals were presented in our previous report[7]. Nominal sensitivity of the optical receivers was $-36 \mathrm{dBm}$ at $1550 \mathrm{~nm}$ and $-37 \mathrm{dBm}$ at $1310 \mathrm{~nm}$. Wavelength-selective couplers and low/high wavelength pass filters were used as wavelength division multiplexers/demultiplexers, and total isolation exceeded $60 \mathrm{~dB}$. To change the direction of the $\lambda_{2}$ signals, we manually changed the fiber connections instead of using optical switches.

Error-free transmission was confirmed in both bi-directional and uni-directional transmission of the $\lambda_{1}$ and $\lambda_{2}$ signals. The bare fiber, wound on bobbins, was set in a temperaturecontrolled chamber and its temperature was varied. The $\tau_{\text {rum }}$ and $\tau_{\text {diff }}$ were measured using time interval counters (Stanford Research Systems; model SR620). Due to the fact that both master and slave were set in the same laboratory, true values of $\tau_{1}$ and $\tau_{2}$ could be directly measured. Under uni-directional transmission conditions, the $\tau_{1}, \tau_{2}$, their difference, $\tau_{\text {diff, }}$, and the chamber temperature are plotted in Figure 5 . The short-term jitter appearing in the $\tau_{\text {diff }}$ data is estimated to be mainly due to the electrical circuit used to derive the time signals from the $156 \mathrm{Mb} / \mathrm{s}$ signals and the resolution of the time interval counters. The variation of $\tau_{1}$ or $\tau_{2}$ was about $74 \mathrm{~ns}$ for a temperature change of $40^{\circ} \mathrm{C}$. The thermal coefficient of the bare-fiber delay, $37 \mathrm{ps} /{ }^{\circ} \mathrm{C} / \mathrm{km}$, obtained from the above results, agrees with the previous reported results $[8]$. The difference in delay, $\tau_{\text {diff }}$, was $113.6 \mathrm{~ns}$ at $25^{\circ} \mathrm{C}$ and the variation of $\tau_{\text {diff }}$ was about $1.2 \mathrm{~ns}$ in this experiment. Under the our experimental conditions in which $\lambda_{1}=1548 \mathrm{~nm}, \lambda_{2}=1315 \mathrm{~nm}, \lambda_{0}=1556 \mathrm{~nm}, \Delta T=40^{\circ} C$, and $L=50 \mathrm{~km}, \tau_{\text {diff } f}$ is $102 \mathrm{~ns}$ and $\Delta \tau_{\text {diff }}$ is 0.98 ns from equations (2) and (3). These calculated values agree with the experimental results. The difference in delay and its variation under these conditions cannot be ignored for sub-nanosecond time transfer and must be measured and compensated for by using the proposed method.

The measured values of $\tau_{1}, \tau_{\text {sum }} / 2$ in the bi-directional transmission and the $\tau_{\text {diff }} / 2$ measured in the uni-directional transmission are plotted against temperature in Figure 6 . The error in determining $\eta_{1}$, which equals the difference between $\tau_{1}$ and the value obtained from equation 
(1), is also plotted. The measured value of $\tau_{1}$ agrees with the delay determined from $\tau_{\text {sum }}$ and $\tau_{\text {diff }}$ within about $0.1 \mathrm{~ns}$ over the entire temperature range. This result shows that subnanosecond time transfer is possible using the proposed method in the $1.31 \mu \mathrm{m}$ and $1.55 \mu \mathrm{m}$ WDM system.

\section{WDM Within $1.55 \mu \mathrm{m}$ Band Transmission}

Distributed feedback (DFB) lasers emitting $+3 \mathrm{dBm}$ of optical power at 1547 and $1546 \mathrm{~nm}$ were used as $\lambda_{1}$ and $\lambda_{2}$ light sources. Two bit-rate signals of $155.52 \mathrm{Mb} / \mathrm{s}$ and $2.48832 \mathrm{~Gb} / \mathrm{s}$, including reference time signals, were tested and were used to directly modulate both DFB-LDs. The sensitivity of the optical receivers was $-36 \mathrm{dBm}$ for $156 \mathrm{Mb} / \mathrm{s}$ signals and $-32 \mathrm{dBm}$ for the 2.488 $\mathrm{Gb} / \mathrm{s}$ signals. The arrayed waveguide gratings, which have 16 channels with a wavelength spacing of $1 \mathrm{~nm}$ in the $1.55 \mu \mathrm{m}$ band, were used as wavelength division multiplexers/demultiplexers ${ }^{[9]}$. The insertion loss was about $7 \mathrm{~dB}$ and the crosstalk with other wavelengths was below $-30 \mathrm{~dB}$. We demonstrated both $156 \mathrm{Mb} / \mathrm{s}$ bi-directional transmission through a $100 \mathrm{~km}$ DSF and 2.488 $\mathrm{Gb} / \mathrm{s}$ bi-directional transmission through a $75 \mathrm{~km}$ DSF while altering the temperature range of the bare DSF wound on bobbins. The DSF had a zero dispersion at the $1561 \mathrm{~nm}$ wavelength. The variation of independently measured delay $\tau_{1}, \tau_{2}$, and their difference, $\tau_{\text {diff }}$, for the 156 $\mathrm{Mb} / \mathrm{s}$ and $2.488 \mathrm{~Gb} / \mathrm{s}$ transmissions are plotted with fiber temperature in Figure 7 and Figure 8 , respectively.

From equation (2), $\tau_{\text {diff }}$ of $100 \mathrm{~km}$ and $75 \mathrm{~km}$ transmissions are $+100 \mathrm{ps}$ and $+76 \mathrm{ps}$ under the experimental conditions. The experimentally measured $\tau_{\text {diff }}$ in Figure 7 and Figure 8 were about $+0.2 \mathrm{~ns}$ and $0.0 \mathrm{~ns}$, respectively. These values agree with the theoretical values within about 0.1 ns. The difference between the experimental and theoretical values is estimated to be caused by the resolution of the time interval counter. Figures $7(\mathrm{~b})$ and $8(\mathrm{~b})$ show the filtered difference in delay, $\tau_{\text {diff }}$, with a time constant of $100 \mathrm{~s}$. The filtered $\tau_{\text {diff }}$ in Figure 7(b) and Figure 8(b) varied within $100 \mathrm{ps}$ and $50 \mathrm{ps}$, respectively. The theoretical value of $\Delta \tau_{\text {diff }}$ due to the temperature change is below $10 \mathrm{ps}$ and it does not appear in Figure $7(\mathrm{~b})$ and Figure $8(\mathrm{~b})$. These results show that the sub-nanosecond delay can be measured using a simple bi-directional transmission in such a close wavelength transmission within the $1.55 \mu \mathrm{m}$ range.

The square root of the Time Variance $[10,11], \sigma_{x}(\tau)$, of the $\tau_{\text {diff }}$, is plotted in Figure 9. The error of the time interval counter (model SR620) is also plotted. The error was measured using the following scheme: the time signal used in the above experiment was split; one was input into the start channel of the counter, and the other was input into the stop channel through a several-meter-long coaxial cable as the proper delay. The $\sigma_{x}(\tau)$ in the averaging time region, $t<100 \mathrm{~s}$, was proportional to $\tau^{-1 / 2}$, which presented white noise phase modulation (PM). In this short term region, the variation of the $\tau_{\text {diff }}$ is estimated to include both the jitter due to the electrical circuit used to derive the time signals and the error of the counter. In particular, the result of the $2.488 \mathrm{~Gb} / \mathrm{s}$ transmission experiment almost coincided with $\sqrt{2}$ times the value of the counter error. This agreement shows the accuracy of the delay measurement in the $2.488 \mathrm{~Gb} / \mathrm{s}$ transmission in the region, $t<100 \mathrm{~s}$, was almost solely restricted by the error of the counter, because the noise was characterized by white noise PM and the tdiff was the 
difference between two values, $\tau_{1}$ and $\tau_{2}$, which were obtained by two independent counters. In the region of $\mathrm{t}>100 \mathrm{~s}, \sigma_{x}(\tau)$ was proportional to $\tau^{0}$ and the variations were characterized by flicker PM. The flicker noises of both the $156 \mathrm{Mb} / \mathrm{s}$ and $2.488 \mathrm{~Gb} / \mathrm{s}$ transmissions were larger than the counter error in the region where $t>1000 \mathrm{~s}$. The causes of these noises are not clear.

\section{CONCLUSION}

An accurate time transfer method using bi-directional WDM signal transmission was proposed. The $1.31 \mu \mathrm{m}$ and $1.55 \mu \mathrm{m}$ signal transmission along a $50 \mathrm{~km}$ fiber was tested and the feasibility of sub-nanosecond time transfer using the proposed method was shown. We also demonstrated the bi-directional dual-wavelength transmission along a $100 \mathrm{~km}$ fiber with a wavelength spacing of $1 \mathrm{~nm}$ in the $1.55 \mu \mathrm{m}$ range. In such a close wavelength transmission system, the subnanosecond delay can be measured using a simple bi-directional dual-wavelength transmission method. These results show that the optical transmission fibers over several tens of kilometers have the capability of time synchronization accuracy of better than $1 \mathrm{~ns}$.

\section{ACKNOWLEDGEMENTS}

The authors wish to thank Dr. Masafumi Koga, Yoshiyuki Hamazumi, Hitoshi Obara, and Dr. Takeshi Kawai of NTT Optical Network Systems Laboratories for their help with the experiments.

\section{REFERENCES}

[1] A. Imaoka, and M. Kihara 1993, "Time signal distribution in communication networks based on synchronous digital hierarchy," Proceedings of the 24th Annual Precise Time and Time Interval (PTTI) Applications and Planning Meeting, 1-3 December 1992, McLean, Virginia, pp. 303-310.

[2] D. Johnson, M. Calhoun, R. Sydnor, and G. Lutes, "A wide-band fiber optic frequency distribution system employing thermally controlled phase compensation," Proceedings of the 24th Annual Precise Time and Time Interval (PTTI) Applicaions and Planning Meeting, 1-3 December 1992, McLean, Virginia, pp. 365-374.

[3] H. Onaka, K. Otsuka, H. Miyata, and T. Chikama 1994, “Measuring the longitudinal distribution of four-wave mixing efficiency in dispersion-shifted fibers," IEEE Photon. Technol. Lett., 6, 1454-1456.

[4] ITU-T Recommendation G. 653, 1993, "Characteristics of a dispersion-dhifted singlemode optical fiber cable."

[5] H. Takahashi, K. Oda, H. Toba, and Y. Inoue 1995, "Transmission characteristics of arrayed waveguide $N \times N$ wavelength multiplexer," IEEE J. Lightwave Technol., 13, 447-455. 
[6] E. Fertein, S. Legoubin, M. Douay, S. Canon, P. Bernage, and P. Niay 1991, "Shifts in resonance wavelength of Bragg gratings during writing or bleaching experiments by UV illumination within germanosilicate optical fibre," Electron. Lett., 27, 1838-1839.

[7] M. Kihara and A. Imaoka 1995, "SDH-based time and frequency transfer system," Proceeedings of the 9th European Frequency and Time Forum (EFTF), Besançon, France, March 1995.

[8] N. Shibata, Y. Katsuyama, Y. Mitsunaga, M. Tateda, and S. Seikai 1983, "Thermal characteristics of optical pulse transit time delay and fiber strain in a single-mode optical fiber cable," Applied Optics, 22, pp. 979-984.

[9] M. Koga, Y. Hamazumi, A. Watanabe, and K. Sato 1995, "Optical path cross-connect systems transport experiment with simulated five-node network," Electron. Lett., 31, 1470-1472.

[10] D.W. Allan, D.D. Davis, J. Levine, M.A. Weiss, N. Hironaka, and D. Okayama 1990, "New inexpensive frequency calibration service from NIST," Proceedings of the 44th Annual Symposium on Frequency Control, 23-25 May 1990, Baltimore, Maryland, pp. 107-116.

[11] D.W. Allan, M.A. Weiss, and J.L. Jespersen 1991, "A frequency-domain view of timedomain characterization of clocks and time and frequency distribution systems," Proceedings of the 45th Annual Symposium on Frequency Control, 29-31 May 1991, Los Angeles, California, pp. 667-678. 


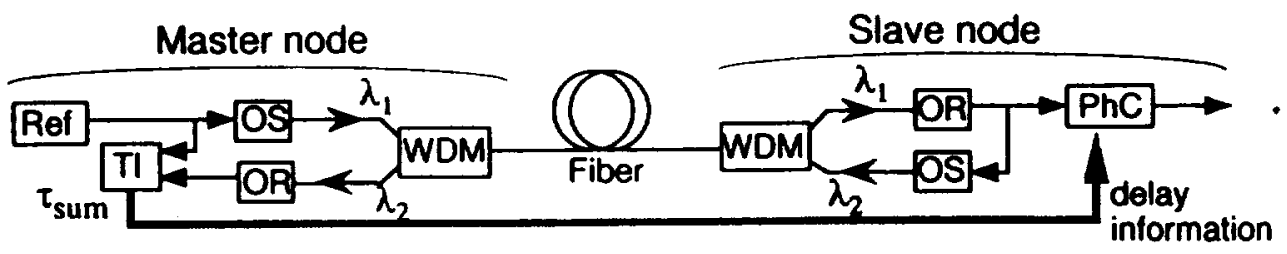

Ref: Reference time generator, PhC: Phase compensator OS: Optical sender, OR: Optical receiver,

WDM: Wavelength division multiplexer / demultiplexer TI: Time interval counter

Fig. I Time transfer method using simple bi-directional dual-wavelength transmission. The delay information is actually transmitted with reference time signals including the $\lambda I$ light.

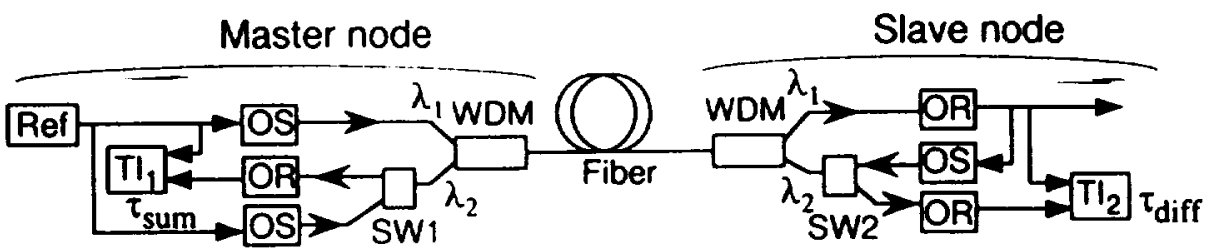

SW: $1 \times 2$ Optical Switch

Fig. 2 Proposed delay measurement scheme. 


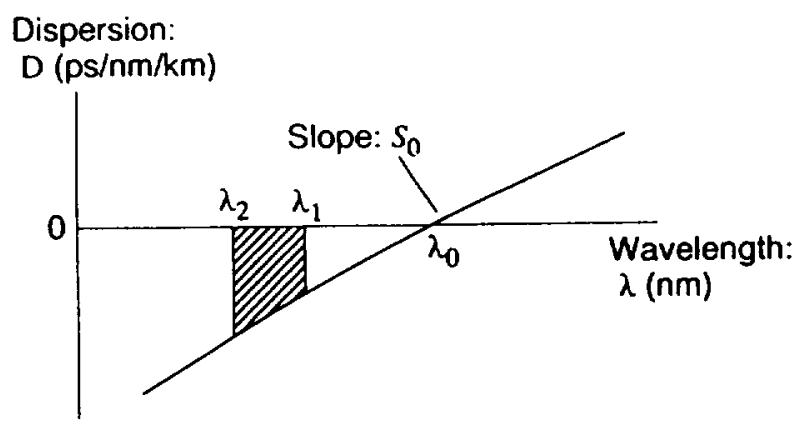

Fig. 3 Chromatic dispersion of optical fiber and its characteristic parameters. The hatched area shows the difference in delay between two wavelengths, $\lambda_{1}$ and $\lambda_{2}$.

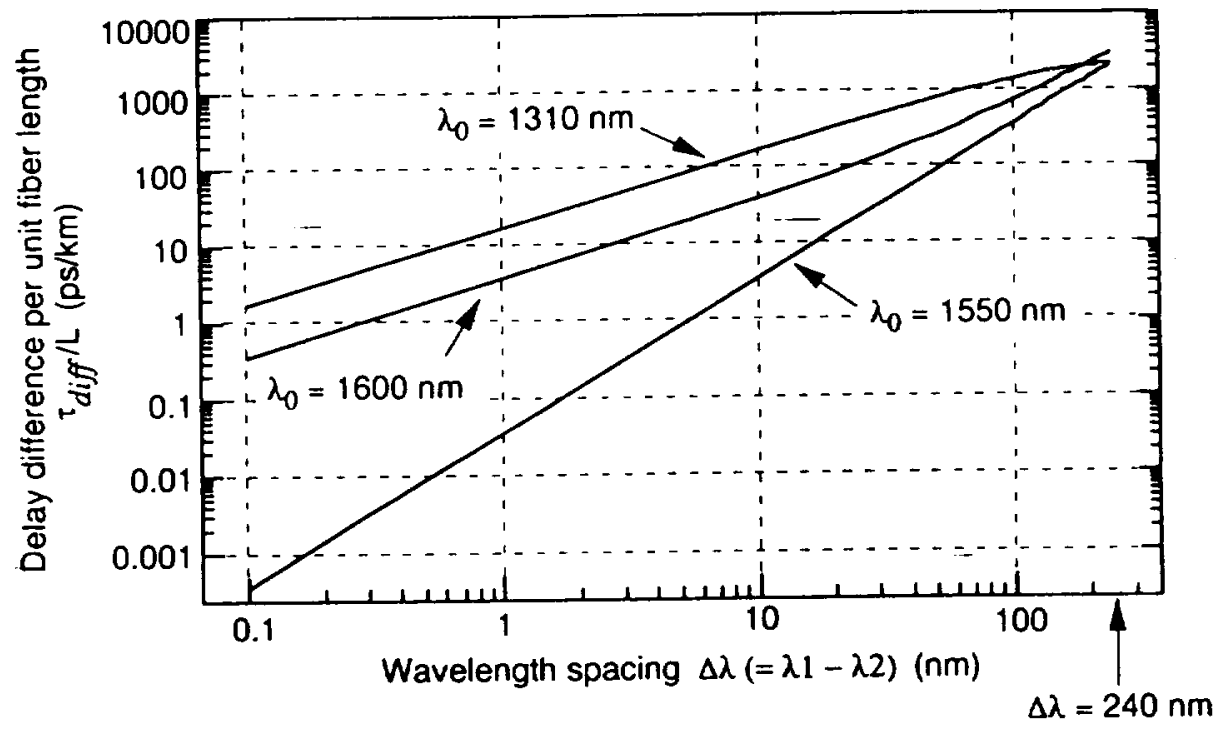

Fig. 4 Delay difference per unit fiber length between two wavelength signals, where the zero dispersion wavelength $\lambda_{0}=1550 \mathrm{~nm}$ and the dispersion slope $S_{0}=+0.07 \mathrm{ps} / \mathrm{nm}^{2} / \mathrm{km}$. 


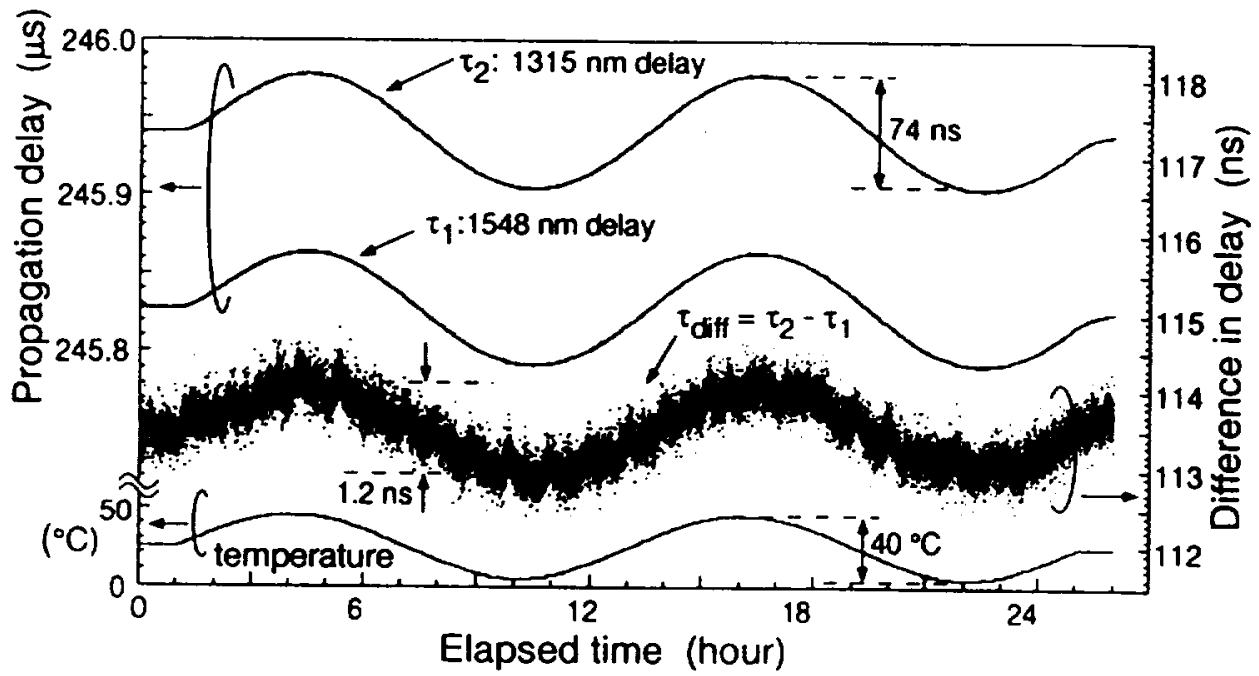

Fig. 5 Measured variations of propagation delay and the difference in delay with temperature change.

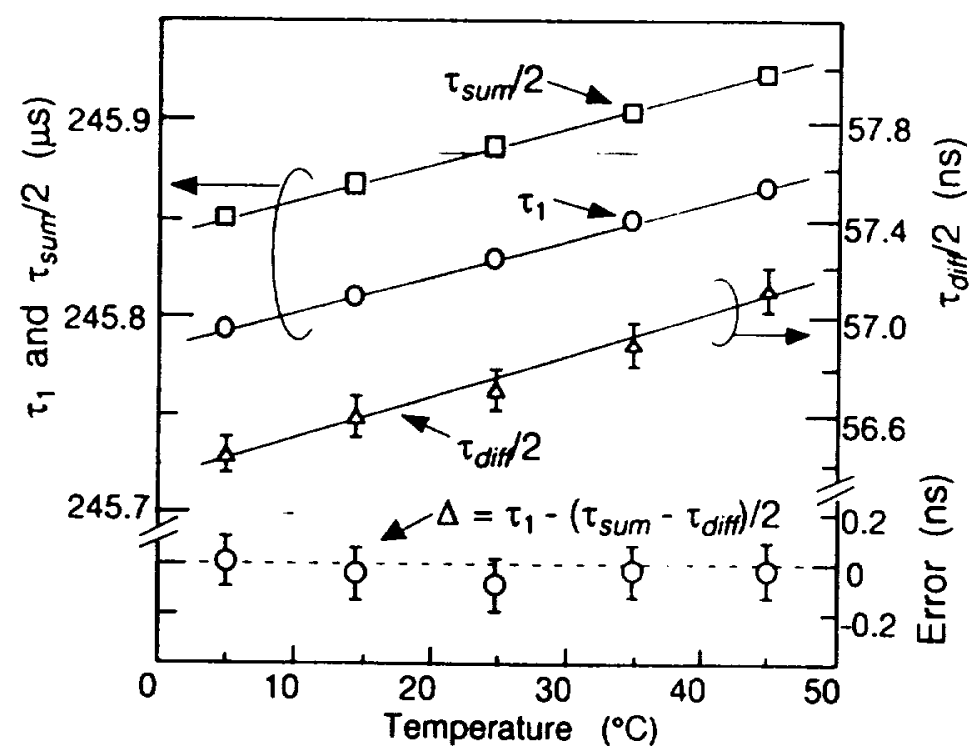

Fig. 6 Measured $\tau_{1}, \tau_{\text {sum }} / 2, \tau_{\text {diff }} / 2$, and the error in determining delay, $\Delta$, with temperature. 


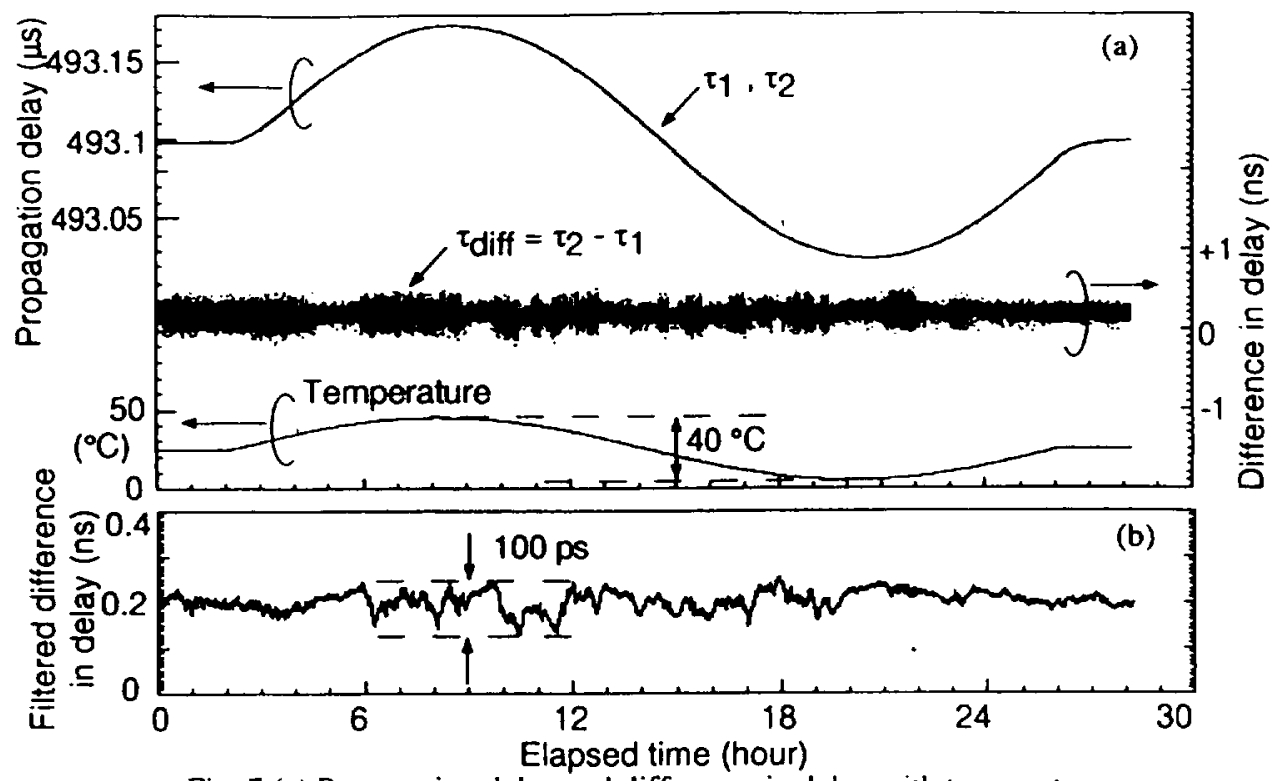

Fig. 7 (a) Propagation delay and difference in delay with temperature.

(b) Filtered difference in delay with time constant of $100 \mathrm{~s}$.

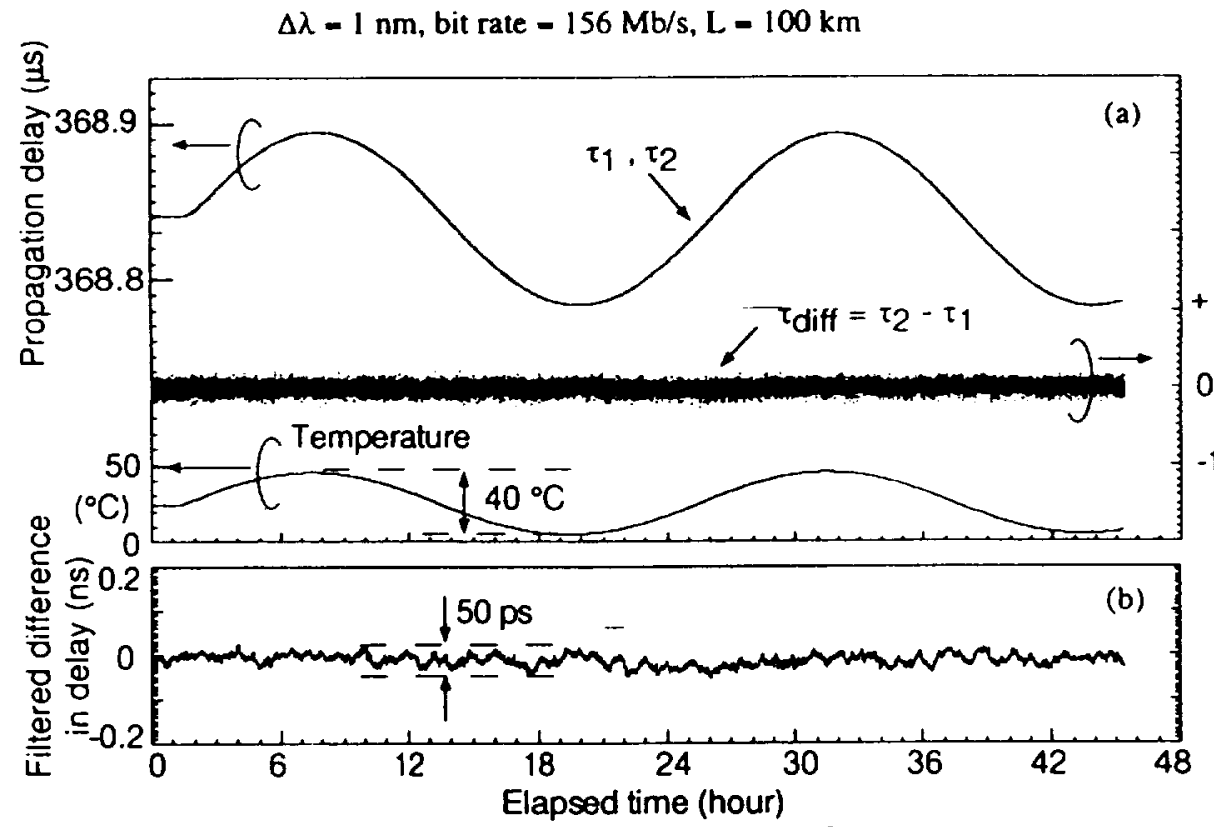

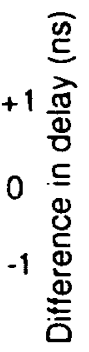

Fig. 8 (a), (b) same as above in conditions of: $\Delta \lambda=1 \mathrm{~nm}$, bit rate $=2.488 \mathrm{~Gb} / \mathrm{s}, \mathrm{L}=75 \mathrm{~km}$ 


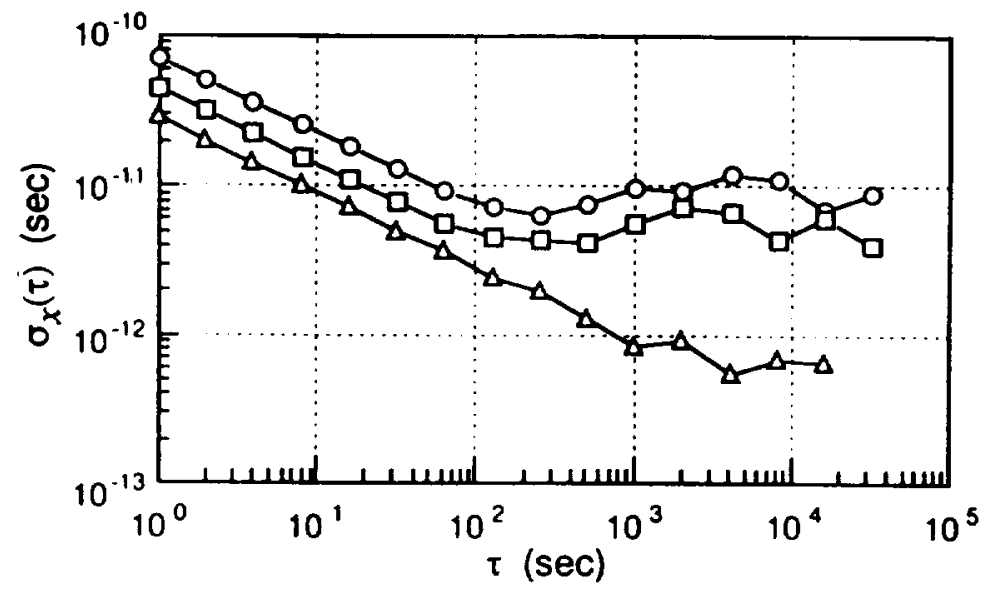

$\longrightarrow \tau_{\text {diff }}$ in $156 \mathrm{Mb} / \mathrm{s}$ 100-km transmission
$\rightarrow-\tau_{\text {diff }}$ in $2.488 \mathrm{~Gb} / \mathrm{s} 75-\mathrm{km}$ transmission
$\rightarrow-$ Counter error

Fig. 9 The square root of Time Variance [11].[12] of $\tau_{\text {diff }}$ compared with the measurement error of the counter. 


\section{Questions and Answers}

DAVID ALLAN (ALLAN'S TIME): If this were extended to longer paths, 1000 kilometers, what would you expect the accuracy to be? Is this extendable to, say, 1000 kilometers?

ATSUSHI IMAOKA (NTT): It depends on the range.

DAVID ALLAN (ALLAN'S TIME): Okay, so 1000 kilometers, what would you expect the error to be for it?

ATSUSHI IMAOKA' (NTT): About 10 nanoseconds. 
